\title{
RÉVOLTES EN ANGLETERRE AU MOYEN ÂGE CENTRAL ET TARDIF
}

Le thème de la révolte - tel que ce recueil d'essais le prouve - jouit d'une popularité traditionnelle parmi les historiens ${ }^{1}$. Les publications des dernières années montrent que les médiévistes, quant à eux, se sont particulièrement intéressés à l'opposition au roi. Ernst Schubert et Frank Rexroth, par exemple, ont analysé en détail les dépositions des rois ${ }^{2}$, tandis que dans une approche plus générale Martin Kintzinger et Jörg Rogge ont publié un recueil d'essais traitant la violence royale et celle exercée contre le roi $^{3}$. Dans leur recueil, la situation en Angleterre est le sujet de l'article de Christopher Allmand, qui examine l'opposition envers le pouvoir royal en Angleterre au Bas Moyen $\hat{A}^{4}{ }^{4}$. À l'époque où il rédigeait cette synthèse (2002/2003), Allmand ne pouvait pas encore consulter l'ouvrage de Claire Valente "The Theory and Practice of Revolt in Medieval England«, publié en $2003^{5}$. Consacrée aux révoltes aristocratiques en Angleterre entre 1215 et 1415, l'étude examine en détail les motivations des rebelles, leurs justifications, leurs statuts sociaux, leurs origines géographiques ainsi que les différentes formes d'actions. Grâce à son analyse, l'anatomie sociale des révoltes aristocratiques est beaucoup mieux connue et ses résultats forment une base très solide pour des recherches ultérieures. Aussi l'analyse suivante profite-t-elle considérablement du travail de Valente, en poursuivant cependant une démarche différente de celle suivi par la chercheuse américaine. Ici, l'intérêt principal porte sur la révolte »comme phénomène révélateur - en tant que moment de cristallisation - de l'évolution

\footnotetext{
${ }^{1}$ Je tiens à remercier Isabelle Deflers, Heidelberg, d'avoir corrigé le français de cet article.

${ }^{2}$ Frank REXROTH, Tyrannen und Taugenichtse. Beobachtungen zur Ritualität europäischer Königsabsetzungen im späten Mittelalter, dans: Historische Zeitschrift 278 (2004), p. 27-53; Ernst SCHUBERT, Königsabsetzung im deutschen Mittelalter. Eine Studie zum Werden der Reichsverfassung, Göttingen 2005 (Abhandlungen der Akademie der Wissenschaften zu Göttingen. Philologisch-Historische Klasse, Folge 3, 267).

${ }^{3}$ Martin KINTZINGER, Jörg ROGGE (dir.), Königliche Gewalt - Gewalt gegen Könige. Macht und Mord im spätmittelalterlichen Europa, Berlin 2004 (Zeitschrift für Historische Forschung, Beiheft 33). Ce recueil d'essais est le résultat d'une section tenue au 44. Deutscher Historikertag (Halle/Saale) en 2002.

${ }^{4}$ Christopher AlLMAND, Opposition to Royal Power in England in the Late Middle Ages, in: KINTZINGER, ROGGE (dir.), Königliche Gewalt - Gewalt gegen Könige (voir n. 3), p. 51-70.

${ }^{5}$ Claire VALENTE, The Theory and Practice of Revolt in Medieval England, Aldershot 2003.
} 
des rapports sociaux en général et de l'incidence du statut social sur la participation au pouvoir en particulier ${ }^{6}$. Par conséquent, l'analyse ne peut pas se limiter aux révoltes aristocratiques, car une étude concentrée sur des questions relatives au statut social du rebelle doit tenir compte de tout type de révolte, qu'elle soit qualifiée d'aristocratique ou de paysanne. Pareillement, il n'est pas possible de se limiter aux seules révoltes »programmatiques «, c'est-à-dire aux révoltes à partir de $1215^{7}$. Bien entendu, les événements autour de la Magna Carta jouent un rôle fondamental dans l'histoire des révoltes anglaises, mais ils ne constituent pas impérativement le point de départ d'une analyse sociale des rebelles et des révoltes. Au contraire, la prise en considération des révoltes avant 1215 donne la possibilité de comparer les révoltes "programmatiques« à celles qualifiées »non-programmatiques« et, par conséquent, d'enrichir la discussion. C'est pour cette raison que la révolte de 1173/1174 est aussi incluse dans l'analyse suivante. Finalement, une telle approche implique l'examen de certains aspects dont le statut social du rebelle au moment de la rébellion n'est qu'un parmi d'autres. L'examen de l'influence du résultat d'une révolte (succès, échec) sur le statut social du rebelle par exemple, ou la comparaison entre la perception du statut du rebelle par ses contemporains et celle de l'historien moderne sont d'autres aspects d'un intérêt considérable. De manière générale, ces questions ouvrent un vaste champ d'enquête, qui, en ce qui concerne aussi l'Angleterre du Haut et Bas Moyen Âge, est loin d'être encore épuisé.

Je vais donc ici tout d'abord discuter la question de savoir qui a le droit ou le devoir de recourir à la révolte et qui ne l'a pas. Dans un deuxième temps, je tenterai de déterminer en quoi le jugement des contemporains et l'analyse des historiens se recoupent à propos de la perception du statut social des acteurs et des meneurs d'une révolte. Dans un troisième temps, j'analyserai brièvement le phénomène d'émergence d'une élite par le biais de la révolte, à savoir si on peut observer une ascension sociale durable d'un groupe ou d'un individu. Finalement, cette étude se consacrera aux conséquences sociales causées par l'échec d'une rébellion. Ici, la question est de déterminer si et en quoi la répression d'une révolte a exacerbé les différences sociales.

L'analyse présente est principalement fondée sur les révoltes de 1173/1174, $1215-1217,1258-1265,1321 / 1322,1381,1387 / 1388$ et 1399 . Une sélection n'est jamais parfaite, mais en couvrant des types divers de révoltes, celle-ci devrait permettre cependant de tirer des conclusions valables.

Avant d'entrer dans le vif du sujet il est nécessaire de signaler que l'usage des termes »rebelle«, »rébellion« et »révolte« n'est pas sans difficulté. Ils désignent en effet des actions menées contre l'ordre existant et ont plutôt une

\footnotetext{
${ }^{6}$ Introduction de Philippe Depreux, p. 9-17, dans ce volume.

${ }^{7}$ VALENTE, Revolt (voir n. 5), p. 10-11.
} 
connotation d'illégitimité que de légitimité ${ }^{8}$. Dans cette étude ces termes sont utilisés dans un sens »neutre«, c'est-à-dire qu'ils sont employés pour désigner toute sorte d'opposition au roi ou au pouvoir royal. La question de savoir, si et quand ces actions ont été considérée comme légitimes ou illégitimes fait partie de la discussion suivante: Est-ce que certains groupes sociaux, contrairement à d'autres, avaient le devoir de révolte? Autrement dit, le statut social d'un rebelle influençait-il sa légitimité?

$\grave{A}$ la fin du XIX ${ }^{e}$ siècle, Frederick Pollock et Frederic W. Maitland ont supposé que, jusqu'en 1352, la prise d'armes contre le roi n'était pas considérée comme une trahison. En 1352, le parlement anglais définit cette action comme trahison et à partir de cette date, le rebelle était en général puni de mort ${ }^{9}$. Aujourd'hui les nouvelles études nous offrent une image plus complexe. Les chercheurs sont d'accord sur le fait qu'en pratique, la révolte était considérée comme crime de trahison dès le règne $\mathrm{d}^{\prime}$ Edouard $\mathrm{I}^{\mathrm{er}}(1272-1307)$; dès la fin du $\mathrm{XIII}^{\mathrm{e}}$ siècle, un rebelle vaincu risquait fortement d'être puni de mort ${ }^{10}$. Mais pour la période comprise entre la conquête normande, en 1066, et le règne d'Edouard I ${ }^{\text {er }}$, les avis sont fortement partagés ${ }^{11}$. Pour les uns, notamment John Gillingham, Matthew Strickland et David Carpenter, l'opposition au roi était toujours considérée comme une trahison; les actions d'Edouard $\mathrm{I}^{\text {er }}$ représentent donc plus une continuité qu'une innovation ${ }^{12}$. Pour les autres, notamment John Bellamy, Maurice Keen et Claire Valente, l'opposition au roi n'était pas considérée comme une trahison avant le règne d'Edouard $\mathrm{I}^{\mathrm{er} 13}$. L'interprétation

${ }^{8}$ Trésor de la langue française. Dictionnaire de la langue du $\mathrm{XLX}^{\mathrm{e}}$ et du $\mathrm{XX}^{\mathrm{e}}$ siècle, $16 \mathrm{t}$, Nancy 1971-1994, t. 14, p. 473-474, 1105-1106.

${ }_{9}^{9}$ Frederick POLLOCK, Frederic W. MAITLAND, The History of English Law, 2 t., Cambridge ${ }^{2} 1968,2$, p. 500-511; The Statutes of the Realm, 11 t., Londres 1810-1828, 1, p. 319-320.

${ }^{10}$ L'importance du règne d'Edouard $\mathrm{I}^{\mathrm{er}}$ dans cette évolution a été soulignée par John $\mathrm{G}$. BELLAMY, The Law of Treason in England in the Later Middle Ages, Cambridge 1970 (Cambridge Studies in English Legal History), p. 11, 14, 23-58; voir aussi Jean DuNBABIN, Government, dans: James H. BURNS (éd.), The Cambridge History of Medieval Political Thought, c. 350-c. 1450, Cambridge 1988, p. 477-519, ici p. 492; VALENTE, Revolt (voir n. 5), p. 33.

"Ibid.

12 John GILLINGHAM, 1066 and the Introduction of Chivalry into England, dans: George GARNETT, John HUDSON (dir.), Law and Government in Medieval England and Normandy. Essays in Honour of Sir James Holt, Cambridge 1994, p. 31-55, en particulier p. 47: „Rebellion was always treason«; Matthew STRICKLAND, Against the Lord's Annointed: Aspects of Warfare and Baronial Rebellion in England and Normandy, 1075-1265, dans: GARNETT, HUDSON (dir.), Law and Government, p. 56-79, ici p. 74; ID., War and Chivalry. The Conduct and Perception of War in England and Normandy, 1066-1217, Cambridge 1996, p. 230-57; David CARPENTER, From King John to the First English Duke, dans: The House of Lords. A Thousand Years of British Tradition, Londres 1994, p. 28-43, 188-193, ici p. 31-35.

${ }^{13}$ BeLlaMY, The Law of Treason (voir n. 10), p. 9-14, 23-58; Maurice KEEN, The Laws of War, Londres 1965 (Studies in Political History), p. 73 et n. 1; ID., Treason Trials under the 
de la clémence des rois envers les rebelles vaincus ou, autrement dit, l'absence d'exécution formelle des rebelles pendant cette période joue un rôle central dans ce débat. Pour Valente, la clémence des rois suggère que, dans le monde aristocratique, se révolter n'était pas considéré comme une trahison ${ }^{14}$. Les partisans de la thèse adverse ont essayé de démontrer, au contraire, que la clémence était surtout le résultat du code chevaleresque introduit par les Normands en 1066 et des circonstances politiques, qui ne permettaient pas de se montrer trop sévère envers les aristocrates rebelles vaincus. Une réponse à ce débat ne peut pas être fournie $\mathrm{ici}^{15}$. En effet, on pourrait ajouter un troisième point de vue, qui envisagerait un compromis entre les deux hypothèses contraires: il est possible d'envisager que, du point de vue royal, une révolte aristocratique était perçue comme une trahison, mais que la clémence répétitive envers les rebelles vaincus fit croire aux aristocrates que leurs actions revêtaient une certaine légitimité. Quoi qu'il en soit, la théorie politique de l'époque n'excluait la possibilité d'une révolte légitime contre le roi. Dans son „Policraticus«, rédigé vers 1159 , Jean de Salisbury écrivait que si le roi agissait comme un tyran, c'est-à-dire s'il ne respectait pas les lois, il devait être combattu (persequitur) ${ }^{16}$. Cependant, Jean et les penseurs qui ultérieurement traitèrent le sujet de la résistance au tyran envisageraient avec beaucoup de précaution la possibilité d'un coup d'État ou même d'un tyrannicide. Clairement, ils ne concevaient cette solution seulement comme une ultima ratio, qu'il fallait mieux éviter ${ }^{17}$.

Law of Arms, dans: ID., Nobles, Knights and Men-at-Arms in the Middle Ages, Londres 1996, n 9, p. 149-166, ici p. 165; VALENTE, Revolt (voir n. 5), p. 32-41.

${ }^{14}$ Ibid.

${ }^{15}$ Il est bien possible que ces controverses trouvent leurs origines aussi dans une interprétation différente du rôle de l'aristocratie dans l'exercice du pouvoir. L'opinion selon laquelle le roi et l'aristocratie exercent ensemble le pouvoir est plus inclinée à attribuer la légitimité à une révolte aristocratique que celle considérant l'aristocratie comme subordonnée au roi. Une analyse détaillée de historiographie fournirait très probablement des éclaircissements sur cet aspect. Pour une analyse de l'historiographie de l'aristocratie de l'Empire, voir le travail de Werner HECHBERGER, Adel im fränkisch-deutschen Mittelalter. Zur Anatomie eines Forschungsproblems, Ostfildern 2005 (Mittelalter-Forschungen, 17).

${ }^{16}$ Clemence C. J. WEBB (éd.), Ioannis Saresberiensis episcopi Carnotensis Policratici sive de nugis curialium et vestigiis philosophorum libri viii, 2 t., Oxford 1909, 1, lib. 3, cap. 15: Et, cum multa sint crimina maiestatis, nullum grauius est eo, quod aduersus ipsum corpus iustitiae exercetur. Tirannis ergo non modo publicum crimen sed, si fieri posset, plus quam publicum est. Si enim crimen maiestatis omnes persecutores admittit, quanto magis illud quod leges premit, quae ipsis debent imperatoribus imperare? Certe hostem publicum nemo ulciscitur, et quisquis eum non persequitur, in seipsum et in totum rei publicae mundanae corpus delinquit. Voir aussi VALENTE, Revolt (voir n. 5), p. 15.

${ }^{17}$ Pour Jean de Salisbury, voir Richard H. RouSE, Mary A. RouSE, John of Salisbury and the Doctrine of Tyrannicide, dans: Speculum 42 (1967), p. 693-709; pour des auteurs ultérieures, voir DUNBABN, Government (voir n. 10), p. 493-496. 
Mais s'il n'y avait vraiment plus aucun espoir que le roi n'améliorât sa conduite, qui avait alors le devoir d'agir contre lui? Jean de Salisbury ne semble pas attribuer ce rôle à un groupe spécifique. Selon lui, quelle que soit la personne (quisquis) qui n'agirait pas contre le tyran, celle-ci commettrait une faute $^{18}$. En effet, la pratique des révoltes montre que la justification selon laquelle »le roi ou le pouvoir royal agit injustement«n'était pas liée à un certain groupe social. Au contraire, c'était une carte blanche jouée par les rebelles, révolte après révolte, indifféremment de leur statut social. Les barons l'utilisaient comme leur justification standard, mais le roi Henri le Jeune, en 1173/1174, ainsi que les rebelles, en 1381 , cherchèrent aussi à en profiter ${ }^{19}$. Cependant, ces deux derniers cas montrent bien que tout le monde ne jouit pas de la même légitimité en tant que rebelle. Henri le Jeune était le fils du roi et ce fait était, au moins du point de vue ecclésiastique, un grave empêchement à toute légitimité de sa cause. Le chroniqueur Raoul de Diceto était tellement choqué par l'action d'Henri le Jeune qu'il inséra dans sa chronique une digression de quelques pages traitant des malheureuses révoltes de fils contre leurs pères à travers l'histoire ${ }^{20}$. Selon Raoul, l'agression du fils contre le père ne pouvait jamais rien provoquer de bon. Il est bien connu que Raoul dans l'ensemble de sa chronique favorisa le vieil Henri ${ }^{21}$, mais son opinion n'est pas un cas isolé. Henri le Jeune lui-même envoya une longue lettre au pape Alexandre III, dans laquelle il cherchait à justifier ses actes qu'il savait violer le commandement fait par Dieu d'honorer ses parents ${ }^{22}$.

La position des paysans et des petits artisans en 1381 était encore beaucoup plus fragile. Bien entendu, en 1381, ce n'était pas la première fois que des

\footnotetext{
${ }^{18}$ Voir n. 16.

19 VALENTE, Revolt (voir n. 5), passim; pour Henri le Jeune, voir sa lettre au pape Alexandre W, Léopold DELISLE et al. (éd.), Recueil des historiens des Gaules et de la France, 24 t., Paris 1869-1904, xvi, p. 643-648. Sur l'authenticité de cette lettre voir Dorothy WHITELOCK et al. (éd.), Councils and Synods with Other Documents Relating to the English Church I. A.D. 871-1204, 2 t., Oxford 1981, 2 (1066-1204), p. 958-959, n. 2; W. J. MILLOR, Christopher N. L. BROOKE (éd.), The Letters of John of Salisbury. II. The Later Letters (1163-1180), Oxford, 1979 (Oxford Medieval Texts), $n^{\circ} 311$, p. 764-765, n. 8.

${ }^{20}$ William StuBBS (éd.), The Historical Works of Master Ralph de Diceto, Dean of London, 2 t., Londres 1876 (Rolls Series, 68), 1, p. 355-366.

${ }^{21}$ John GillinghaM, Royal Newsletters, Forgeries and English Historians: Some Links between Court and History in the Reign of Richard I, dans: Martin AURELL (dir.), La cour Plantagenêt (1154-1204). Actes du colloque tenu à Thouars du 30 avril au 2 mai 1999, Poitiers 2000 (Civilisation médiévale, 8), p. 171-186, ici p. 178-179.

${ }^{22}$ Delisle et al. (éd.), Recueil des historiens des Gaules et de la France (voir n. 19), xvi, p. 644: Novimus, Pater venerande, admirari quamplures, et etiam detestari tam gravem, peccatis nostris exigentibus, inter nos et dominum patrem meum dissensionem obortam, cum Dominus, sicut jamjam a pluribus didicimus, de honorandis parentibus speciale mandatum dederit, et quod ipsos persequentes ignominiosi erunt et infelices. Igitur, ne, de humani temeritate judicii, in nostrae famae depressionem tam inhumana, tam atrox in nos culpa refundatur, nostrae dissensionis causam apostolatui vestro paucis intimare necessarium duximus.
} 
paysans participaient à des révoltes ${ }^{23}$. L'action la plus célèbre était peut-être celle des paysans de Peatling Magna (Leicestershire) pendant la révolte de 1258-1265. Quelques jours après la bataille d'Evesham (4 août 1265), des habitants du village de Peatling Magna essayèrent d'arrêter le chariot et les chevaux menés par un homme de Peter de Neville, partisan d'Henri III. Peter de Neville rapportait qu'ils étaient, lui-même et ses hommes, accusés de trahison et d'autres crimes sous prétexte qu'ils avaient porté atteinte au bien-être de la communauté du royaume ainsi qu'aux barons ${ }^{24}$. Mais à Peatling Magna comme ailleurs avant 1381 , les actions des groupes sociaux inférieurs se déroulaient toujours au cours de révoltes initiées et menées par des barons. Le fait que des paysans soient eux-mêmes meneurs d'une révolte était une chose inattendue et inacceptable ${ }^{25}$ : les groupes dirigeants de la société, l'aristocratie et le clergé, n'accordaient pas aux paysans le devoir et, par conséquent, le droit d'agir comme gardiens du royaume. Les chroniqueurs, alors même qu'ils montraient une certaine compréhension pour la fureur des paysans contre les taxes imposées par le parlement ${ }^{26}$, toléraient beaucoup moins leurs actions armées contre le roi ou leurs seigneurs. ${ }^{27}$ Le plus radical parmi eux était Thomas Walsingham, moine du grand monastère de Saint-Alban; son dégoût pour les actions des rustici apparaît à chaque page du récit qu'il fait des événements

${ }^{23}$ VALENTE, Revolt (voir n. 5), p. 68-162.

${ }^{24}$ Henry G. RICHARDSON, George O. SAYLES (éd.), Select Cases of Procedure without Writ under Henry III, Londres 1941 (The Publications of the Selden Society, 60), $\mathrm{n}^{\circ} 30:$ Et Petrus venit et duxit predictos obsides et defendit vim et iniuriam quando etc., et bene cognoscit quod predicti Galfridus, Simon et alii sunt in custodia sua tanquam obsides pro fine viginti marcarum quam homines de predicta villa ipsi et hominibus suis fecerunt in transitu eorum per eandem villam post predictum conflictum, inponentes ipsi et hominibus suis seditiones et alia opprobria, eo quod fuerunt contra utilitatem communitatis regni et contra barones, ac homines suos verberauerunt, vulnerauerunt et maletractauerunt. David CARPENTER, English Peasants in Politics, 1258-1267, dans: Past \& Present 136 (1992), p. 3-42, ici p. 3.

${ }^{25}$ Voir aussi Richard E. BARTON, Lordship in the County of Maine, c. 890-1160, Woodbridge 2004, p. 166-167.

${ }^{26}$ Voir, par exemple, Geoffrey H. MARTIN (éd.), Knighton's Chronicle 1337-1396, Oxford 1995 (Oxford Medieval Texts), p. 206-208, par exemple p. 208: Cumque uidissent communes de Cancia et finitimis locis erga eos rem sic grauiter peragi et noua quasi importabilia onera eis indesiniter absque remedio imponi, istiusmodi oppressionis grauamen ulterius ferre non ualentes, conferebant adinuicem quam remedii uel subsidii cautelam inde reperire ualerent. Vivien $\mathrm{H}$. GALBRAITH (éd.), The Anonimalle Chronicle, 1333 to 1381. From a Ms. Written at St. Mary's Abbey, York, Manchester 1970, p. 133-135.

${ }^{27}$ MARTIN (éd.), Knighton's Chronicle (voir n. 26), p. 208-220, par exemple, p. 210: Sicque congregate sunt [Commons] super le Blakeheth, ubi pre multitudine seipsos obliti et causa pristine non contenti, necque minoribus sceleribus pacati, maiora inexquisita mala meditate sunt inmisericorditer, nec a suo nephando proposito desistere disposuerunt, quoadusque omnes procures regni et magnates funditus exstirparent. GALBRAITH (éd.), The Anonimalle Chronicle (voir n. 26), p. 135-150; Chris GIVEN-WILSON (éd.), The Chronicle of Adam Usk, 1377-1421, Oxford 1997 (Oxford Medieval Texts), p. 2-5. 
de 1381. Pour Thomas, témoin direct de la révolte ${ }^{28}$, le comportement des rebelles était absolument inacceptable. Sa description des événements qui se déroulèrent en la tour de Londres exprime toute son indignation: „Les rebelles qui, avant, comptaient parmi les serfs les plus humbles, entraient et sortaient comme des seigneurs; et des porchers se plaçaient au dessus des soldats, même s'ils ne se comportaient pas comme des chevaliers, mais comme des paysans ${ }^{29}$. La description du comportement de Wat Tyler, le meneur des rebelles, pendant les négociations avec le roi Richard II à Smithfield est tout aussi comparable. Comme précédemment dans la tour de Londres, le rebelle avait la prétention de se comporter comme un seigneur: Tyler, selon Walsingham, déclara que le chevalier John Newton, intermédiaire entre le roi et lui, devrait s'approcher de lui-même, alors qu'il était en selle, à pied plutôt qu'à cheval. Newton, de son côté, qui, comme Walsingham le remarque avec plaisir, "n'avait pas encore complètement oublié son vieil honneur chevaleresque«, répondit qu'étant donné que Tyler était assis sur un cheval, ce n'était pas lui faire une insulte si lui-même l'approchait aussi assis sur un cheval. Cette réponse n'était pas à même de calmer la situation et quand, par la suite, Tyler commença à menacer Newton avec son couteau, le roi commanda au maire de Londres, William Walworth, de l'arrêter. En exécutant cet ordre, Walworth et ses hommes attaquèrent le meneur des rebelles avec leurs épées. Mortellement blessé, Tyler tomba de son cheval ${ }^{30}$. Dans ce contexte, la description de cette chute est beaucoup plus qu'un simple détail. Elle a une signification symbolique: le paysan n'était pas un seigneur. Il ne faisait pas partie de l'aristocratie et n'avait pas le droit d'assumer un rôle aristocratique.

Or, c'était l'aristocratie, et en particulier les grands barons, qui s'était appropriée le rôle de corriger les actions du roi et qui y trouvait une certaine reconnaissance. Les magnats se considéraient comme les conseillers naturels du roi. Pour eux, donner consilium n'était pas seulement un devoir prescrit par

${ }^{28}$ Pour Thomas Walsingham, voir John TAYLOR et al. (éd.), The St Alban's Chronicle. The Chronica maiora of Thomas Walsingham, i (1376-1394), Oxford 2003 (Oxford Medieval Texts), p. xviii-xxvii; ID., English Historical Literature in the Fourteenth Century, Oxford 1987 , p. 60-77, 319-320.

${ }^{29}$ ID. et al. (éd.) The St Alban's Chronicle (voir n. 28), p. 424: Intrabant et exibant ut domini, qui quondam fuerant uilissime condicionis serui; et preferebant se militibus non tam militum, set rusticorum subulci.

${ }^{30}$ Ibid., p. 436-438: Ille [Wat Tyler] quidem indignatus qui equo uectus miles accesserat, et non pedes, mox cum furore dixit, decere eum pocius pedes quam equum insidens sue presencie propinquare. Miles vero, nondum oblitus omnino milicie sue ueteris probitatis, ilico sic respondit, ,Sedenti, inquit, stibi in equo non est iniuriosum me equitum accessisser. [...] At mox ab aliis ministris regiis circumclusus, gladiis diuersis in locis corporis transuerberatur; corruensque de equo in terram, primum dedit spem Anglicane milicie, fere mortue, communibus posse resisti.; voir aussi Siméon LuCÉ et al. (éd.), Chroniques de J. Froissart, 15 t., Paris 1869-1975, x (1380-1382), p. 119-122. 
leur hommage au roi, mais aussi un droit $^{31}$. Le roi devait les consulter. S'il ne le faisait pas et, par conséquent, prenait des décisions injustes, les magnats croyaient avoir l'obligation de prendre des mesures correctives. Bien entendu, il serait naif de croire que leurs motifs étaient d'ordre altruiste. Les ambitions personnelles ont toujours joué un rôle important et, parfois, elles ont même dominé les actions des barons. La justification classique invoquée par les rebelles, selon laquelle le roi était entouré de mauvais conseilleurs qu'il fallait éloigner, n'était pas seulement une excuse protectrice pour éviter de se voir accusé de vouloir attaquer le roi personnellement ${ }^{32}$, mais reposait souvent sur de fortes rivalités de cour ${ }^{33}$. Néanmoins, il semble que le bien de la communauté n'était pas uniquement une formule vide pour cacher des ambitions personnelles. Les actions autour de la Magna Carta laissent deviner une responsabilité pour la communauté du royaume au moins parmi quelques barons ${ }^{34}$ et un demi siècle plus tard, pendant la révolte de 1258-1265, les rebelles firent constamment référence au bien de la communauté comme but principal de leur action $^{35}$. Ces deux exemples montrent bien qu'en pratique, il est très difficile de déterminer dans quels cas un tel argument est utilisé par conviction ou comme simple prétexte. On peut même se demander si une telle distinction est valable, car du point de vue d'un baron, la poursuite de ses propres ambitions n'était pas nécessairement incompatible avec la poursuite du bien de la communauté. Robert Fitz Walter, par exemple, l'un des meneurs de la révolte contre le roi Jean, avait de forts intérêts personnels ${ }^{36}$ et les historiens ont peutêtre raison de ne pas le compter parmi les rebelles principalement intéressés par le bien de la communaute ${ }^{37}$. Il est toutefois imaginable que pour Robert et ses partisans, le titre de »maréchal de l'armée de Dieu et de la Sainte Église en

${ }^{31}$ Gavin LANGMUIR, Per commune consilium regni in Magna Carta, dans: Studia Gratiana 15 (1972), p. 465-485; voir aussi James C. HOLT, Magna Carta, Cambridge ${ }^{2} 1992$, p. $317-$ 321; pour l'Empire, voir Bernd SCHNEIDMÜLLER, Konsensuale Herrschaft. Ein Essay über Formen und Konzepte politischer Ordnung im Mittelalter, dans: Paul-Joachim HeINIG et al. (dir.), Reich, Regionen und Europa in Mittelalter und Neuzeit. Festschrift für Peter Moraw, Berlin 2000 (Historische Forschungen, 67), p. 53-87.

${ }^{32}$ Joel T. ROSENTHAL, The King's "Wicked Advisers« and Medieval Baronial Rebellions, dans: Political Science Quarterly 82 (1967), p. 595-618; VALENTE, Revolt (voir n. 5), p. 40.

${ }^{33} \mathrm{La}$ révolte de $1387 / 1388$ en est un exemple, voir ibid., p. 171-187.

${ }^{34}$ HOLT, Magna Carta (voir n. 31), p. 520-522; Ralph TURNER, Magna Carta. Through the Ages, Harlow 2003, p. 57; VALENTE, Revolt (voir n. 5), p. 60, 65.

${ }^{35}$ Voir par exemple Reginald E. TREHARNE, Ivor J. SANDERS, Documents of the Baronial Movement of Reform and Rebellion, 1258-1267, Oxford 1973 (Oxford Medieval Texts), p. 98 (Provisions of Oxford): [...] ad provisionem istam sic factam pro communi utilitate tocius regni.; cf, ibid., p. 92; VALENTE, Revolt (voir n. 5), p. 78-90.

${ }^{36}$ Sidney PAINTER, The Reign of King John, Baltimore 1949, p. 31-35; James C. HOLT, The Northerners. A Study in the Reign of King John, Oxford 1965, p. 78-83, 96-97.

${ }^{37}$ Voir par exemple TURNER, Magna Carta (voir n. 34), p. 56. 
Angleterre « (marescallus exercitus dei et sancte ecclesie in Anglia ${ }^{38}$ ), qu'il portait pendant la révolte, était bien plus qu'un titre prétentieux. Un exemple illustrant l'inséparable lien entre les intérêts personnels et altruistes de façon beaucoup plus explicite que chez Robert Fitz Walter est celui de Simon de Montfort. John Maddicott a démontré qu'au cours des événements de 12581265 , Simon poursuivait à la fois ses intérêts personnels en même temps qu'il œuvrait à une réforme du royaume - en particulier celle de la justice - pour le bien de la communauté. Les deux, ambition et idéalisme, étaient le moteur de ses actions ${ }^{39}$.

Outre le rôle du baron comme conseiller du roi, les révoltes aristocratiques bénéficiaient d'un certain air de légitimité dû au fait que prendre les armes pour résoudre un conflit faisait partie de la culture aristocratique ${ }^{40}$. Dans ce contexte il est important de rappeler que l'épée était le symbole du statut aristocratique et qu'elle distinguait ainsi l'aristocratie des autres groupes de la société $^{41}$. Donc, même si la fonction d'homme de guerre en soi ne légitimait pas une révolte aristocratique, une telle action paraissait beaucoup moins extraordinaire qu'une révolte menée par de simples paysans.

En outre, la question de la légitimité d'une révolte est peut-être liée à celle du traitement de l'adversaire. Ici, on observe un changement net entre le Moyen Âge central et le Moyen Âge tardif: l'adversaire est traité avec une dureté croissante. Au Moyen Âge central, les rebelles hésitaient à faire la guerre contre la personne du roi. Au lieu de cela, l'agression militaire se dirigeait surtout vers les partisans du roi ${ }^{42}$. Et même si une rencontre militaire directe entre roi et rebelles avait lieu, la vie du roi ne semble pas avoir été soumise à un danger particulier ${ }^{43}$. En 1264, par exemple, pendant la bataille de Lewes entre Henri III, ses fils Edouard et Henri d'un côté et Simon de Montfort de l'autre, le but des rebelles n'était probablement pas de tuer le roi ou ses fils $^{44}$. Aussi le fait que Simon de Montfort, vainqueur de la bataille, ne déposa pas Henri III suggère que c'était encore une chose de lever les armes contre le

${ }^{38}$ HOLT, Magna Carta (voir n. 31), p. 490-491 (appendix II); voir aussi Henry R. LUARD (éd.), Matthaei Parisiensis, monachi sancti Albani, chronica majora, 7 t., Londres 1872-1883 (Rolls Series, 57), t. 2, p. 586: Quae cum magnates cognovissent, constituerunt Robertum filium Walteri principem militiae suae, appellantes eum marescallum exercitus Dei et sanctae ecclesiae.

${ }^{39}$ John MADDICOTT, Simon de Montfort, Cambridge 1994, p. 151-345, en particulier p. 151-191.

${ }^{40}$ STRICKLAND, War and Chivalry (voir n. 12), p. 98-131; Richard W. KAEUPER, Chivalry and Violence in Medieval Europe, Oxford 1999, p. 129-188; BARTON, Lordship in the County of Maine (voir n. 25), p. 146-173.

${ }^{41}$ David CROUCH, The Image of Aristocracy in Britain, 1000-1300, Londres 1993, p. 190198.

${ }^{42}$ STRICKLAND, Against the Lord's Annointed (voir n. 12), p. 57-70.

${ }^{43}$ Ibid., p. 73.

${ }^{44}$ Ibid. 
roi, et tout à fait une autre de le déposer. Néanmoins, le traitement d'Henri III suite à la bataille de Lewes semble avoir été le premier signe d'un durcissement du traitement de l'adversaire tel qu'on l'observe au XIV ${ }^{e}$ siècle. Simon de Montfort n'a pas seulement pris le contrôle du gouvernement du royaume, mais aussi de la personne du roi dont il contrôlait en effet chaque mouvement. L'humiliation que ce traitement occasionna pour le roi fut bien remarquée par les contemporains ${ }^{45}$. Un siècle plus tard, un roi captif risquait fortement d'être déposé et même de perdre la vie. En 1386, après l'échec du roi Richard II à respecter les stipulations d'un compromis conclu avec les Commons en 1385, un groupe de magnats menés par l'oncle du roi, Thomas, duc de Gloucester, alla chercher le roi pour l'obliger à expulser ses conseilleurs, qui ne donnaient rien qu'un ineptum consilium. S'il ne les expulsait pas, il risquerait sa déposi$\operatorname{tion}^{46}$. Un an plus tard, déçu des actions de Richard, les magnats mirent à exécution leur menace. Ils emprisonnèrent le roi et, voyant qu'il persistait à ignorer leurs requêtes, ils le déposèrent. Après trois jours »sans couronne« Richard céda et fut restitué dans sa position royale ${ }^{47}$. Une douzaine d'années plus tard, il fut moins chanceux. Après une décennie de relative tranquillité concernant les relations entre Richard et les magnats, le roi décida en 1397 de punir les rebelles de 1387/1388. La condamnation du duc de Gloucester, de l'earl de

${ }^{45}$ MADDICOTT, Simon de Montfort (voir n. 39), p. 270-345, en particulier p. 280-287, 311, 331, 337-338. À Evesham en 1265, Simon de Montfort a mené Henri, armée aux couleurs de Simon, dans la bataille, Harry ROTHWELl (éd.), The Chronicle of Walter of Guisborough previously Edited as the Chronicle of Walter of Hemingford or Hemingburgh, Londres 1957 (Camden Series, 89), p. 201. Cette action a été interprétée différemment par les historiens. Matthew Strickland a voulu y voir une tentative de Simon de faire tuer Henri par ses propres hommes, tandis que Björn Weiler a suggéré que la présence du roi servait à légitimer les actions et les troupes de Simon, STRICKLAND, Against the Lord's Annointed (voir n. 12), p. 73; Björn WEILER, Symbolism and Politics in the Reign of Henry III, dans: Michael PRESTWICH et al. (dir.), Thirteenth Century England IX. Proceedings of the Durham Conference 2001, Woodbridge 2003, p. 15-41, ici p. 29.

${ }^{46}$ MARTIN (éd.), Knighton's Chronicle (voir n. 26), p. 353-360, p. 360: Set et unum aliud de nuncio nostro [les magnats] superest nobis ex parte populi uestri [Richard II] uobis imitare. Habent enim ex antiquo statuto, et de facto non longe retroactis temporibus experienter (quod dolendum est habito), si rex ex maligno consilio quocumque uel inepta contumacia aut contemptu seu proterua uoluntate singulari, aut quouis modo irregulari se alienauerit a populo suo, nec uoluerit per iura regni et statuta ac laudabiles ordinaciones cum salubri consilio dominorum et procerum regni gubernari et regulari, set capitose in suis insanis consiliis propriam uoluntatem suam singularem proterue exercere, extunc licitum est eis cum communi assensu et consensu populi regni ipsum regem de regali solio abrogare, et propinquiorem aliquem de stirpe regia loco eius in regni solio sublimare [...]; voir aussi L. C. HeCTOR, Barbara D. HARVEY (éd.), The Westminster Chronicle, 1381-1394, Oxford 1982 (Oxford Medieval Texts), p. 226-229.

${ }^{47}$ Maude V. ClarKe, Vivian H. GalBraITH, The Deposition of Richard II, dans: Bulletin of the John Rylands Library 14 (1930), p. 125-181, ici p. 157-161, 157: [...], adierunt [rebelles] regem Ricardum et eum de solio regali deposuerunt et sic per triduum mansit discoronatus; VALENTE, Revolt (voir n. 5), p. 174-175. 
Warwick et celle de l'earl d'Arundel ne provoquait aucune une résistance notable, mais la confiscation des biens du duc de Lancaster après la mort de John of Gaunt en 1399 provoqua une nouvelle révolte ${ }^{48}$. Le fils de John of Gaunt, Henri Bolingbroke, n'accepta pas la confiscation de son héritage et leva les armes contre le roi. Sa campagne fut victorieuse. Il emprisonna Richard en la tour de Londres, le fit déposer et se fit couronner à sa place. Richard mourut peu de temps après en prison ${ }^{49}$.

Dans un deuxième temps, on constate que le traitement réservé par le roi aux rebelles a suivi un déroulement similaire. Au Moyen Âge central, un rebelle aristocrate vaincu pouvait, dans la plupart des cas, compter sur la clémence du roi. Le comportement d'Henri II après sa victoire triomphale sur ses fils en 1173/1174 est un exemple célèbre de la clémence royale. Le roi n'a pas puni ses fils, mais il s'est réconcilié avec eux. Henri le Jeune dut accepter de transmettre à son frère, Jean, quelques châteaux et revenus en Angleterre, en Normandie et en Anjou, mais son statut de successeur d'Henri II ne fut pas remis en question. En ce qui concerne les partisans du jeune Henri et ses frères, on s'accorda à rétablir le status quo ante, c'est-à-dire la situation qui existait quinze jours avant le commencement des hostilités ${ }^{50}$. Cette clémence ne peut pas cacher le fait que la révolte était une entreprise pleine de risques comme en témoignent les cas du roi d'Ecosse, Guillaume, de Ranulf, earl de Chester, et de Robert, earl de Leicester. Ces trois hommes, qui agirent de manière particulièrement virulente contre Henri II, étaient volontairement exclus

${ }^{48}$ Ibid., p. $195-196$.

${ }^{49}$ GIVEN-WILSON (éd.), Adam Usk (voir n. 27), p. 50-91, ici p. 68; Sentencia deposicionis. Et sic - ut quid mora? - licet seipsum [Richard] deposuerat ex habundanti, ipsius deposicionis sentencia, in scriptis redacta, consensu et auctoritate totius parliementi per magistrum Iohannem Treuar de Powysia, Assaven' episcopum, palam publice et solempniter lecta fuit ibidem. Successio novi regis. Et sic, uacante regno, consensu totius parliementi dictus dux Lancastr' in regem erectus, per archiepiscopos predictos in sede regali ad statim intronizari optinuit. Ibid., p. 88-90: Mors Ricardi. Iam hii in quibus Ricardus nuper rex fiduciam habuit releuaminis ceciderunt, qua audito, magis usque ad sui mortem lugendo condoluit, in castro de Pomffret, catenis ligato et uictualium penuria, domino N. Swynford ipsum tormentante, sibi ultimo die Februarii miserabiliter contingentem; VALENTE, Revolt (voir n. 5), p. 196200; Anthony TUCK, Richard II and the English Nobility, Londres 1973, p. 187-225; Nigel SAUL, Richard II, Yale 1997, p. 405-434.

${ }^{50}$ Léopold DELISLE, Élie BERGER (éd.), Recueil des actes de Henri II, roi d'Angleterre et duc de Normandie, concernant les provinces françaises et les affaires de France, 3 t., Paris 1916-1927 (Chartes et diplômes relatifs à l'histoire de France), 2, $\mathrm{n}^{\circ} 468$ : Notum sit tam presentibus quam futuris quod pax inter dominum H[enricum] regem Angl[orum] et filios ejus, H[enricum] videlicet regem et Ricardum et Gaufridum in hunc modum Deo volente reformata est. [...] Et dominus rex debet rehabere, ipse et barones et homines sui, omnes terras suas et castella sua, que habuerunt $X V$. diebus antequam filii sui recederent $a b$ eo. Similiter barones et homines sui qui recesserunt ab eo et secuti sunt filios suos, debent rehabere terras suas quas habuerunt $X V$. diebus antequam ipsi ab eo recederent; voir aussi ibid., $\mathrm{n}^{\circ} 488$. 
des termes du traité conclu entre Henri et ses fils, à Falaise en avril $1174^{51}$. En ce qui les concerne, la révolte leur coûta très cher. Henri II confisqua les terres de Ranulf et Robert jusqu'en $1177^{52}$, tandis que Guillaume dut accepter en sa qualité de roi d'Ecosse de voir son rang abaissé du fait qu'il fut obligé de prêter hommage à Henri pour son royaume ${ }^{53}$. En 1174, le traitement sévère d'un rebelle était encore exceptionnel, mais au cours du siècle suivant la sévérité devint la norme: ce n'est plus une réconciliation à laquelle le rebelle vaincu devait s'attendre, mais à une exécution. Là aussi, on observe encore une fois un premier signe de durcissement de comportement au cours des événements de 1258-1265. À la bataille décisive d'Evesham en 1265, les troupes royales ne tentèrent pas de capturer les meneurs de l'opposition. Au contraire, Edouard et l'earl de Gloucester décidèrent de les tuer ${ }^{54}$. Il est possible que cette mesure radicale ait été provoquée par le traitement d'Henri et ses fils après la bataille de Lewes. Il est possible également que cette décision ait été prise parce qu'ils n'avaient pas encore les moyens judiciaires de faire exécuter Simon de Montfort après la bataille $e^{55}$. De toute façon, ils voulaient mettre un terme au règne de Simon et, comme la mort brutale de celui-ci le suggère, faire un exemple: tué lors de la bataille, son corps fut mutilé par ses adversaires: la tête, les bras, les pieds et les testicules furent coupés ${ }^{56}$. Une mort turpissima et

${ }^{51}$ Delisle, Berger (éd.), Actes de Henri II (voir n. 50), 2, n 468: Prisones vero qui cum domino rege finem fecerunt ante pacem factam, videlicet rex Scocie, et comes Legrecestrie, et comes Cestr[ie], et Radulfus de Fulgeriis, et obsides eorum, et obsides aliorum prisonum quos prius habuerat, sunt extra conventionem istam. Raoul de Fougères était un autre adversaire éminent d'Henri II. Mais contrairement aux trois autres, Raoul a réussi à établir rapidement de bonnes relations avec Henri II, Judith A. EVERARD, Brittany and the Angevins. Province and Empire, 1158-1203, Cambridge 2003 (Cambridge Studies in Medieval Life and Thought), p. 54-55.

${ }^{52}$ William STUBBS (éd.), Gesta regis Henrici secundi Benedicti abbatis: the Chronicle of the Reigns of Henry I and Richard I, A.D. 1169-1192, Known Commonly under the Name of Benedict of Peterborough, 2 t., Londres 1867 (Rolls Series, 49), 1, p. 134: Cumque rex audisset eum [Robert of Leicester] ita pie loquentem, commotus pietate, reddidit ei omnia tenementa sua, in integrum, sicut ea habuit quindecim diebus ante gwerram, [...]; p. 135: In eodem concilio apud Northamtun rex reddidit comiti Cestriae totam terram suam, quam habuit ante guerram; sed retinuit in misericordia sua castellum Cestriae.

${ }^{53}$ DeLisLe, BERgER (éd.), Actes de Henri II (voir n. 50), 2, $n^{\circ} 470-471, \mathrm{n}^{\circ}$ 470: Willelmus rex devenit homo ligius domini regis contra omnem hominem de Scotia et de omnibus aliis terris suis, etc [...].

${ }^{54}$ Olivier DE LABORDERIE et al., The Last Hours of Simon de Montfort: a New Account, dans: English Historical Review 115 (2000), p. 378-412, ici p. 403.

${ }^{55}$ Ibid.

${ }^{56}$ Annales monasterii de Oseneia (A.D. 1016-1347), dans: Henry R. LUARD (éd.), Annales Monastici, 5 t., Londres, 1864-1869 (Rolls Series, 36), iv, p. 1-352, ici p. 170-171: Et cum tantae multitudini diu resistere non praevaleret, se ipsum et causam suam Deo et gloriosae Matri suae commendans, ibidem interfectus est morte turpissima et inaudita. Nam caput ejus amputabant, manus, pedes, et membra virilia, quod dictu horribile est, abscidebant, et super ea sicut super arma sua ut quis quid tolleret, quasi sortem mittebant. 
inaudita comme les annales d'Oseney la commente ${ }^{57}$, mais une mort qui n'était pas sans rappeler la punition des traîtres par castration ${ }^{58}$. Pour les hommes d'Henri III, Simon était un traître et devait être puni comme tel ${ }^{59}$. C'était encore un événement isolé, mais Edouard semble avoir appris la leçon. Probablement aussi influencé par le droit romain, qui accordait le droit de prendre les armes uniquement aux princes sans souverain, Edouard introduisit pendant son règne la pratique de traiter les rebelles comme des traîtres, c'està-dire de les condamner à mort ${ }^{60}$. Sous le règne de son fils, Edouard II, cette pratique devint la norme. Le meneur de la révolte de 1321/1322, Thomas, duc de Lancaster, et certains parmi ses alliés en firent l'expérience: après leur défaite et leur arrestation à Boroughbridge en 1322 , le procès que leur fit le roi les accusait entre autre de trahison. Déclaré coupables, ils furent exécutés ${ }^{61}$. Enfin, en 1352, cette règle fut fixée par écrit lorsque le parlement qualifia la prise d'armes contre le roi de crime de trahison ${ }^{62}$.

Tournons-nous maintenant vers la comparaison entre la perception du statut social des rebelles par leurs contemporains et celle de l'historien moderne. La question de savoir en quoi le jugement des contemporains et celui des historiens se recoupent est avant tout un problème de sources. Pour le Moyen Âge central au moins, les opinions contemporaines se trouvent principalement dans les chroniques. S'il existe une abondance d'autres sources concernent les participants à la révolte, l'historien a la possibilité de comparer et par conséquent d'arriver à des conclusions différentes de celles du chroniqueur. Mais si la chronique est la seule source, les moyens de l'historien sont plus restreints. Il ne peut qu'espérer que le chroniqueur mentionne un grand nombre de partici-

${ }^{57}$ Voir note précédente.

${ }^{58}$ Sur la castration comme punition du crime de trahison, voir Klaus VAN EICKELS, Domestizierte Maskulinität. Die Integration der Normannen in das westfränkische Reich in der Sicht des Dudos von St-Quentin, dans: Ingrid BENNEWITZ, Ingrid KASTEN (dir.), Genderdiskurse und Körperbilder im Mittelalter. Eine Bilanzierung nach Butler und Laqueur, Münster 2002 (Bamberger Studien zum Mittelalter, 1), p. 97-134.

${ }^{59}$ Un argument évoqué aussi par WEILER, Symbolism and Politics in the Reign of Henry III (voir n. 45), p. 29-30.

${ }^{60}$ Bellamy, The Law of Treason (voir n. 10), p. 1-58, en particulier p. 14; pour le royaume de France, voir Simon H. CUTTLER, The Law of Treason and Treason Trials in Later Medieval France, Cambridge 1981 (Cambridge Studies in Medieval Life and Thought), p. 4-27.

${ }^{61}$ Friedrich W. D. BRIE (éd.), The Brut or the Chronicles of England, 2 t., Londres 1906/1908 (The Early English Text Society 131, 136), 1, p. 221-224; Wendy R. CHILDS (éd.), Vita Edwardi Secundi. The Life of Edward the Second, Oxford 2005 (Oxford Medieval Texts), p. 210215; selon l'auteur anonyme de la »Vita Edwardi secundi«, le roi appelait ses adversaires des proditores (traitres), p. 204; voir aussi Annales Paulini, dans: William STUBBS (éd.), Chronicles of the Reigns of Edward I and Edward II, 2 t., Londres 1882/1883 (Rolls Series, 96), 1, p. 253-370, 302-303; Gesta Edwardi de Canarvan, auctore canonico Bridlingtoniensi, cum continuatione ad A.D. 1377, dans: STUBBS (éd.), Chronicles of the Reigns of Edward I and Edward II, 2, p. 23-151, ici p. 76-77.

${ }^{62}$ Voir p. 169. 
pants afin de pouvoir, à travers des études prosopographiques, accepter ou rejeter son jugement. La révolte de 1173/1174 est un exemple d'un événement connu principalement grâce aux chroniques ${ }^{63}$. Bien informés, les chroniqueurs fournissent de longues listes de participants. L'un d'entre eux, Roger of Howden, le rédacteur probable de la $» G e s t a$ regis Henrici « ${ }^{64}$, avait même probablement accès à une liste provenant de l'administration royale fournissant les noms des rebelles ${ }^{65}$. Les chroniqueurs nous offrent l'image d'une rébellion contre le roi Henri II, qui fut initiée par des membres de sa famille, notamment par son fils aîné, Henri le Jeune. Ils nous informent qu'Henri le Jeune trouva des appuis auprès du roi de France, Louis VII, du roi d'Écosse, Guillaume, et auprès des membres de tous les rangs de l'aristocratie en Angleterre comme en Normandie. En plus de leur rang, les chroniqueurs ont aussi observé d'autres aspects du statut social des aristocrates rebelles. Ils nous indiquent que des barons établis et des iuvenes, c'est-à-dire des jeunes chevaliers qui n'avaient pas encore établi leur propre seigneurie, participaient à la révolte ${ }^{66}$. Les historiens modernes ont largement accepté l'image donnée par les chroniqueurs. La révolte de 1173/1174 compte aujourd'hui parmi les exemples de révolte aristocratique menée par un membre de la famille royale ${ }^{67}$.

Les sources plus nombreuses concernant les révoltes ultérieures permettent une analyse plus fine et moins dépendante des chroniqueurs, mais de manière

${ }^{63}$ Par exemple, Chronica Roberti de Torigneio, abbatis monasterii Sancti Michaelis in Pericolo Maris, dans: Richard HowleTT (éd.), Chronicles of the Reigns of Stephen, Henry II and Richard I, 4 t., Londres 1884-1889 (Rolls Series, 82), iv, p. 255-267; Ronald C. JOHNSTON (éd.), Jordan Fantosme's Chronicle, Oxford 1981; STUBBS (éd.), Diceto (voir n. 20), 1, p. 371-397; ID. (éd.), Gesta regis (voir n. 52), 1, p. 42-79; ID. (éd.), Chronica Rogeri de Hovedene, 4 t., Londres 1868-1871 (Rolls Series, 51), 2, p. 45-82.

${ }^{64}$ David CORNER, The Gesta Regis Henrici Secundi and Chronica of Roger, Parson of Howden, dans: Bulletin of the Institute of Historical Research 56 (1983), p. 126-144.

${ }^{65}$ STUBbs (éd.), Gesta regis (voir n. 52), 1, p. 45-46, 56-57; STRICKLAND, Against the Lord's Annointed (voir n. 12), p. 58.

${ }^{66}$ Pour les barons établis, voir STUBBS (éd.), Diceto (voir n. 20), 1, p. 371: Johannes siquidem comes de Soeneis, Hugo comes Cestrensis, Robertus comes de Mellent, Jocelinus Crispin, Gilebertus de Tiveleres, Robertus de Monte Forti, Willelmus Patricius, Hasculfus de Sancto Hylario, Radulfus dominus Fulgeriarum, et plures mediae manus homines, quos ex justis et probabilibus causis rex pater exhaeredaverat, regis filii cesserunt in partem, non quia causam ejus iustiorem attenderent, sed quia rex pater, regiae titulos dignitatis ampliare procurans, superborum et sullimium colla calcaret, castella patriae suspecta vel everteret vel in suam redigeret potestatem; pour les iuvenes, voir Chronica Roberti de Torigneio (voir n. 63), iv, p. 255-256, 260, pour la citation suivante, voir p. 255-256: In quadragesima, quia rex Henricus removerat a consilio et famulatu filii sui. Asculfum Sancto Hylario et alios equites juniores, ideo ille [Henri le Jeune] iratus recessit a patre, [...]. Sur les iuvenes en général, voir Georges DUBY, Dans la France de Nord-Ouest. Au XII ${ }^{e}$ siècle: les rjeunes la société aristocratique, dans: Annales. E. S. C. 29 (1964), p. 835-846.

${ }^{67}$ Robert BARTLETT, England under the Norman and Angevin Kings, 1075-1225, Oxford 2000, p. 54-56; Daniel POWER, The Norman Frontier in the Twelfth and Early Thirteenth Centuries, Cambridge 2004 (Cambridge Studies in Medieval Life and Thought), p. 398-401. 
générale, il semble que l'historien moderne n'aboutisse pas à des conclusions substantiellement différentes de celles des contemporains. Les travaux de Sydney Painter et de James C. Holt, par exemple, ont beaucoup amélioré notre connaissance relative aux participants de la révolte de $1215-1217^{68}$. En ce qui concerne les chevaliers, l'historien moderne est certainement beaucoup mieux renseigné sur l'identité et les motifs des rebelles que les chroniqueurs, mais les deux chercheurs ont aussi confirmé l'opinion répandue à l'époque, selon laquelle les meneurs de la révolte contre Jean sans Terre étaient des barons, en particulier des barons du nord et de l'est du royaume ${ }^{69}$. Il en va de même pour la révolte de 1381. Les chroniques de l'époque rédigées aux grandes abbayes de Saint-Alban, Westminster, Bury Saint-Edmund ou Evesham qualifient les paysans de principaux agents de la révolte. Thomas Walsingham, moine de Saint-Alban, décrit les rebelles dans le comté d'Essex comme étant des rustici namque, quos natiuos vel bondos uocamus, simul cum ruralibus accolis in Estsexia $^{70}$. D'autres sources font référence aussi aux petits artisans ou parlent

${ }^{68}$ PAINTER, The Reign of King John (voir n. 36), p. 284-289; HOLT, The Northerners (voir n. 36); ID., Magna Carta (voir n. 31), p. 123-87, 294.

${ }^{69}$ Voir par exemple William STUBBS (ẻd.), Memoriale fratris Walteri de Coventria, 2 t., Londres 1872/1873 (Rolls Series, 58), 2, p. 217: Dissensio orta est inter Johannem regem Angliae et quosdam de proceribus pro scutagio quod petebat ab illis qui non ierant nec miserant cum ipso in Pictaviam. Dantibus enim illud plurimis, contradixerunt ex Aquilonaribus nonnulli, illi videlicet qui anno praeterito regem ne in Pictaviam transiret impedierunt: [...]; p. 219: Igitur in hebdomada Paschali convenerunt in manu valida ex condicto apud Stamforde, et quoniam ex Aquilonaribus partibus pro parte majori venerant, vocati sunt adhuc Aquilonares. Voir aussi Joseph STEVENSON (éd.), Radulphi de Coggeshall chronicon Anglicanum, Londres 1875 (Rolls Series, 66), p. 170-185; Annales prioratus de Dunstaplia (A.D. 1-1297), dans: LUARD (éd.), Annales Monastici (voir n. 56), 3, p. 1-408, ici p. 43; Francisque MICHEL (éd.), Histoire des ducs de Normandie et des rois d'Angleterre, Paris 1840 (Société de l'histoire de France), p. 145, 147-148, 160; pour les documents royaux, voir HoLT, The Northerners (voir n. 36), p. 11-12. James C. Holt a souligné l'aspect intéressant selon le terme shomme du nord « (Northerner) pour décrire les rebelles n'était pas toujours utilisé pour le même groupe de personnes. Les chroniqueurs bien informés ont distingué entre les barons du nord qualifiés d'hommes du nord et les autres rebelles, tandis que d'autres chroniqueurs l'employaient pour décrire tous les rebelles. Vers le début de 1215 la chancellerie royale semble avoir commencé à utiliser également ce terme pour décrire tout rebelle quelque soit leur origine géographique, ibid., p. 8-16.

${ }^{70}$ TAYLOR et al. (éd.), The St Alban's Chronicle (voir n. 28), p. 410; HeCTOR, HARVEY (éd.), The Westminster Chronicle (voir n. 46), p. 2; voir aussi Thomas ARNOLD (éd.), Memorials of St. Edmund's Abbey, 3 t., Londres 1890-1896 (Rolls Series, 96), 3, p. 125-126; Rodney Hitron, Bond Men Made Free. Medieval Peasant Movements and the English Rising of 1381, Londres 1973, p. 176-177; concernant l'image de la révolte de 1381 dans les chroniques anglaises et françaises, voir aussi Neithard BULST, )Jacquerie( und )Peasants' Revolt ` in der französischen und englischen Chronistik, dans: Hans PATZE (dir.), Geschichtsschreibung und Geschichtsbewußtsein im späten Mittelalter, Sigmaringen 1987 (Vorträge und Forschungen, 31), p. 790-819; Frederik W. N. HuGENHOLTZ, Drie boerenopstanden uit de veertiende eeuw. Vlanderen, 1323-1328, Frankrijk, 1358, Engeland, 1381, s'Gravenhage ${ }^{2} 1978$, p. $150-157$. 
plus généralement des Commons ${ }^{71}$. Même si le terme de "révolte de paysans «, qui date du dernier quart du XIX ${ }^{e}$ siècle, est encore en usage aujourd'hui ${ }^{72}$, les chercheurs ont appris à distinguer plus finement les différents groupes de participants: les paysans et les petits artisans (surtout dans les villes) constituent les deux groupes principaux, auxquels viennent s'ajouter aussi des prêtres ou des membres de la basse aristocratie de campagne (minor gentry) ${ }^{73}$. Donc, plutôt que de contredire les témoignages des contemporains, les chercheurs les précisent. Aujourd'hui comme à l'époque, la révolte de 1381 est considérée principalement comme une révolte des groupes de niveau social inférieur, dans laquelle les paysans, surtout dans les campagnes, jouèrent un rôle important.

Le dernier point de cette analyse des révoltes anglaises est consacré au rôle de la révolte comme catalyseur d'un changement au sein de la hiérarchie sociale. La révolte a-t-elle causé l'émergence d'une élite? Observe-t-on une ascension sociale durable des rebelles? Ici la réponse est ambivalente: En ce qui concerne l'individu, le cas de Henri Bolingbroke démontre qu'une ascension sociale durable était possible, même si celle-ci signifiait plutôt l'exception que la norme. Henri, duc, devint roi d'Angleterre en 1399. Ce fut grâce à sa révolte qu'il reçut la couronne et il eut l'occasion de fonder la maison royale de Lancaster $^{74}$. Henri présente donc un cas d'ascension sociale au plus haut niveau possible.

Mais ce phénomène ne s'était pas étendu à un groupe d'individus; les révoltes anglaises n'ont pas produit de nouvelles élites ${ }^{75}$. Certes, on pourrait se de-

${ }^{71}$ MARTIN (éd.), Knighton's Chronicle (voir n. 26), p. 208: [...] communes de Cancia et finitimis locis [...]; Annales prioratus de Dunstaplia (voir n. 69), p. 415-417; CLARKE, GALBRAITH, The Deposition of Richard II (voir n. 47), p. 164, 166; HILTON, Bond Men Made Free (voir n. 70), p. 177.

${ }^{72}$ Richard B. DOBSON, The Peasants' Revolt of 1381, Londres 1970, p. 13; Neithard BULST, Wacquerie und >Peasants' Revolts (voir n. 70), p. 793, n. 25, qui renvoie comme point de reference à Richard B. DOBSON, Remembering the Peasants' Revolt, 1381-1981, dans: William H. LIDDELL, Robert G. WOOD (dir.), Essex and the Great Revolt of 1381. Lectures Celebrating the Six Hundredth Anniversary, Chelmsford 1982. Des termes alternatifs, utilisés, par exemple, par Paul Freedman dans sa contribution à la New Cambridge Medieval History, sont "English Rising" (»le soulèvement anglais") ou simplement "la révolte de 1381 «, Paul FreEDMAN, Rural Society, dans: Michael JonES (dir.), The New Cambridge Medieval History, VI. c. 1300-c. 1415, Cambridge 2000, p. 82-101, ici p. 98-101.

${ }^{73}$ Hilton, Bond Men Made Free (voir n. 70), p. 176-185; ID., Soziale Programme im englischen Aufstand von 1381, dans: Peter Blickle (dir.), Revolte und Revolution in Europa. Referate und Protokolle des internationalen Symposiums zur Erinnerung an den Bauernkrieg 1525 (Memmingen, 24.-27. März 1975), München 1975 (Historische Zeitschrift, Beiheft 4), p. 31-46, ici p. 30-34; DoBSON, The Peasants' Revolt (voir n. 72), p. 13-14.

${ }^{74}$ Henri était un parent de Richard II, mais il n'était pas le premier en ligne à lui succéder, GIVEN-WILSON (éd.), Adam Usk (voir n. 27), p. xviii-xix.

${ }^{75}$ Examinant la révolte de 1321/1322 Scott Waugh a montré comment la basse aristocratie de campagne (minor gentry) a essayé d'en tirer profit. Les actions étaient largement dirigées contre les concurrents de même niveau social. Même si individus ont réussi à améliorer leur position, le groupe quant à lui ne bénéficia d'aucune ascension sociale durable, Scott WAUGH, 
mander si le conseil de 25, instauré par la clause 61 de la Magna Carta $^{76}$, ou encore si le conseil de 24 , ensuite de 15 et finalement de 12 membres ( 3 électeurs et 9 conseillers) mis en place pour gouverner aux côtés d'Henri III en $1258-1265^{77}$, n'avait pas la capacité de générer une nouvelle élite. De telles spéculations reposent sur des bases très incertaines. En effet, la durée des fonctions de ces conseils était trop brève pour laisser des traces durables dans la hiérarchie sociale. Parmi les révoltes considérées ici, celle de 1381 avait le potentiel le plus important pour provoquer un changement dans la hiérarchie sociale. Le désir de voir le servage aboli semble avoir été une revendication commune: et toutz crierent a une voice qils ne vodroient aler avaunt qils avoient les traitours deinz la Toure et chartres destre free de toutz maners de servage et des autres maners des poyntes qils vodroient demander, relate l'auteur de l'»Anonimalle Chronicle ${ }^{78}$. Ne voyant pas d'autre solution Richard accepta les demandes des rebelles et leurs concéda des chartes qui les libérèrent du servage ${ }^{79}$. En plus de l'abolition du servage, circulait également l'idée de supprimer l'aristocratie et de répartir la terre de façon égalitaire. Il ne devait plus exister qu'un roi et des hommes libres devant tous être du même rang. Le même principe devait être appliqué à l'organisation de l'Église. Seul un évêque ou un archevêque devait être à la tête de l'Église, et toute la hiérarchie entre cet évêque et le prêtre paroissial devait être abolie. En outre, l'Église ne devait plus agir comme seigneur foncier ${ }^{80}$. Si toutes ces idées avaient été mises en œuvre, les conséquences politiques et sociales auraient été radicales. Mais les rêves des paysans furent de courte durée. Après la mort de leur meneur, Wat Tyler, la révolte fut rapidement étouffée et leurs chartes de liberté, annulées ${ }^{81}$. La hiérarchie sociale ancestrale restait en place. La révolte de 1381 n'eut donc pas d'effets immédiats sur l'ordre social; la révolte ne catalysa pas la progression sociale durable d'un groupe. À long terme cependant, il est possible que la révolte ait contribué au lent processus de dissolution du servage en Angleterre ${ }^{82}$.

The Profits of Violence: the Minor Gentry in the Rebellion of 1321-1322 in Gloucestershire and Herefordshire, dans: Speculum 52 (1977), p. 843-869.

${ }^{76}$ HOLT, Magna Carta (voir n. 31), p. 468-473.

${ }^{77}$ MADDICOTT, Simon de Montfort (voir n. 39), p. 157-60, 285-88.

${ }^{78}$ GaLBRAITH (éd.), The Anonimalle Chronicle (voir n. 26), p. 143.

${ }^{79}$ TAYLOR et al. (éd.), The St Alban's Chronicle (voir n. 28), p. 440-442: Tenor carte extorte per uim a domino rege talis extat, mutatis nominibus communitatum, prout diversis comitatibus mittebantur. Carta regis de manumissione rusticorum: [...] Sciatis quod de gracia nostra speciali manumisimus uniuersos ligeos et singulos subditos nostros et alios comitatus Hertfordie, et ipsos et eorum quemlibet, ab omni bondagio exuimus, et quietos facimus per presentes [...]. Cf. également GalBRAITH (éd.), The Anonimalle Chronicle (voir n. 26), p. 143.

${ }^{80}$ HILTON, Bond Men Made Free (voir n. 70), p. 224-230.

${ }^{81}$ LUCÉ et al. (éd.), Les chroniques de J. Froissart (voir n. 30), x (1380-1382), p. 123; HILTON, Bond Men Made Free (voir n. 70), p. 224.

${ }^{82}$ FREEDMAN, Rural Society (voir n. 72), p. 100-101 Pour une opinion plus pessimiste, voir DOBSON, Peasants' Revolt (voir n. 72), p. 27. 
Comme les révoltes ne servirent pas de catalyseur à une quelconque ascension sociale, leur répression n'exacerba pas non plus, en général, les différences sociales d'une manière signifiante. Certes, en 1174, Guillaume, roi d'Écosse avait dû subir une dégradation lorsqu'il prêta hommage à Henri II pour son royaume ${ }^{83}$. Mais quelques années plus tard, en 1189, Richard I lui restitua son indépendance ${ }^{84}$. De même, les événements de 1381 n'eurent pas de conséquences majeures dans ce domaine. Bien entendu, la punition des rebelles fut dure. Les meneurs furent exécutés, et la ville de Bury Saint-Edmund et 287 personnes furent exclues de l'amnistie générale accordée par le Parlement de novembre/décembre $1381^{85}$. Mais en comparaison de la répression brutale de la Jacquerie (1358) en France, la façon dont le roi d'Angleterre, l'aristocratie et le clergé étouffèrent la révolte fut moins sanglante ${ }^{86}$. De même, les vainqueurs renoncèrent, de façon générale, à aggraver les conditions de vie des paysans et petits artisans ${ }^{87}$.

À l'exception de la révolte de 1381 , toutes les révoltes dirigées contre le roi ou le pouvoir royal entre 1066 et 1400 étaient d'origine aristocratique ou, au moins, menées par des barons. Se révolter était donc surtout une affaire d'aristocrates. L'aristocratie, en particulier les magnats, par sa fonction, qui définissait aussi son statut social, s'appropriait le droit - le devoir - de recourir aux armes afin de corriger la conduite d'un roi tyrannique ou inutilis. Elle y trouvait une certaine reconnaissance; une reconnaissance, cependant, qui semble avoir été relative plutôt qu'absolue. Le rebelle aristocrate se voyait accorder plus de tolérance qu'un rebelle paysan, mais on peut cependant douter du fait que le roi accordait vraiment à l'aristocrate un droit à se rebeller. Edouard $\mathrm{I}^{\text {er }}$ et ses successeurs ne le firent pas, et il est bien possible qu'ils ne fissent que reprendre une attitude déjà adoptée par leurs prédécesseurs.

L'action des réformateurs ou des rebelles n'influença pas la structure sociale d'Angleterre d'une manière significative. Une révolte pouvait changer le statut social d'un individu, mais elle ne semble pas avoir transformé celui d'un groupe. Bien entendu, les révoltes aristocratiques n'avaient pas comme but, en général, de changer la hiérarchie - c'était d'ailleurs plutôt le contraire. Mais si on considère le fait que le résultat d'une action ne dépend pas nécessairement d'une intention déterminée, cette constance de l'ordre social est néanmoins frappante. Elle mérite de faire l'objet des études ultérieures.

\footnotetext{
${ }^{83}$ Voir p. 178-179.

84 John GILLINGHAM, Richard I, Yale 1999, p. 113.

${ }^{85}$ DOBSON, Peasants' Revolt (voir n. 72), p. 325-333.

${ }^{86}$ SAUL, Richard II (voir n. 49), p. 73-75; BULST, >Jacquerie n. 70), p. 800 .

${ }^{87}$ Il est possible qu'au niveau politique, la révolte de 1381 ait attribué, comme Claire Valente l'a proposé, au processus d'éloignement entre la haute aristocratie et les couches inférieures de la société, cf. VALENTE, Revolt (voir n. 5), p. 170.
} 